\title{
Accelerating consensus of self-driven swarm via adaptive speed
}

\author{
Jue Zhang $^{a}$, Yang Zhao ${ }^{a}$, Baomei $\operatorname{Tian}^{a}$, Liqian Peng $^{a}$, \\ Hai-Tao Zhang ${ }^{b, c}$, Bing-Hong Wang ${ }^{a}$, Tao Zhou ${ }^{a, d}$ \\ a Department of Modern Physics and Nonlinear Science Center, University of \\ Science and Technology of China, Hefei Anhui, 230026, PR China \\ ${ }^{b}$ Department of Engineering, University of Cambridge, Cambridge CB2 1PZ, \\ U.K. \\ ${ }^{c}$ Department of Control Science and Engineering, Huazhong University of Science \\ and Technology, Wuhan 430074, PR China \\ ${ }^{d}$ Department of Physics, University of Fribourg, Chemin du Muse 3, CH-1700 \\ Fribourg, Switzerland
}

\begin{abstract}
In resent years, Vicsek model has attracted more and more attention and been well developed. However, the in-depth analysis on the convergence time are scarce thus far. In this paper, we study some certain factors that mainly govern the convergence time of Vicsek model. By extensively numerical simulations, we find the convergence time scales in a power law with $r^{2} \ln N$ in the noise-free case, where $r$ and $N$ are horizon radius and the number of particles. Furthermore, to accelerate the convergence, we propose a new model in which the speed of each particle is variable. The convergence time can be remarkably shortened compared with the standard Vicsek model.
\end{abstract}

Key words: Vicsek model; self-driven swarm; convergence time; adaptive speed

\section{Introduction}

In nature, collective motion of large numbers of organisms is one of the most ubiquitous biological phenomena, from motion of groups of ant [1], colonies of bacteria [2] and cells [3] in a small scale, to migration of flocks of birds and

Email address: zhutou@ustc.edu (Tao Zhou $\left.{ }^{a, d}\right)$. 
schools of fish [4] in a large scale. Those different forms of collective behaviors can only be understood by considering the very number of large interactions among group members. Studies on this issue is significant. On one hand, we can deepen our understanding of such collective behaviors; on the other hand, we could probably extract some generic rules from those natural systems, which can be applied to other relevant realms, such as the control of unmanned vehicles or robots [5].

Inspired by biological collective motion, Vicsek et al. [6] described each individual in the collective motion as an self-driven particle moving with a constant speed and adjusting the direction according to the average direction over neighborhood. By this means, each particle will achieve the same velocity through finite steps [7], namely a kinetic phase transition from disorder to order state $[6,8,9]$. Besides, many modified models about self-driven swarm are also proposed. For example, Couzin et al. [10,11] proposed a more biologically realistic model, and studied the effect of the repulsion, alignment, attraction and leadership on swarm formation.

In a word, though its simplicity, the Vicsek model is of great academic significance [12], which has become a theoretical templet for the consensus of swarm. However, previous works mostly focus on the analysis of steady state, while they pay little attention on the time used to achieve that steady state. As a new aspect of investigating the collective motion, the convergence time also has great significance to be explored. On one hand, it can be a criterion of evaluation of a consensus strategy. On the other hand, detailed discussions on the relationship between the factors and the convergence time can provide us some insights about the collective dynamics. Therefore, in this paper, we will study which factors influence the convergence time and how can a group of agents get to be coherent as quickly as possible. First, we will discuss the relationship between the convergence time and the particle density as well as the horizon radius of individuals. Then, considering the fact that the speed of an individual in biological group or removable robots usually can vary in a certain range, we abandon the strong assumption in the Vicsek model, namely, the constant speed. Instead, we propose a swarm model with variable speed, upon which a new communication protocol is designed, and the convergence time under this protocol is shorter than that of the standard Vicsek model.

\section{Convergence time in the Vicsek model}

A group of $N$ particles are considered which are moving in an $L \times L$ square with periodic boundary conditions. In the Vicsek model, the particles are moving in an identical constant speed but different directions, with the initial conditions that the particles are randomly distributed in the square, and their 
initial moving directions are uniformly distributed in the interval $[-\pi, \pi)$. At each time step, the direction of each particle is determined by the average direction of the velocity of all the particles (including itself) within the circle of horizon radius $r$ centered the given particle. Mathematically speaking, the position of the $i$ th particle is updating according to:

$$
\vec{x}_{i}(t+\delta t)=\vec{x}_{i}(t)+\vec{v}_{i}(t) \delta t
$$

while the corresponding discrete presentation is:

$$
\vec{x}_{i}(t+1)=\vec{x}_{i}(t)+\vec{v}_{i}(t) .
$$

And its direction is updating as:

$$
\theta_{i}(t+1)=\left\langle\theta_{i}(t)\right\rangle_{r}+\Delta \theta_{i}
$$

where $\Delta \theta_{i}$ denotes the noise, and $\left\langle\theta_{i}(t)\right\rangle_{r}$ denotes the average direction of all the particles within the horizon radius $r$, including the $i$ th particle itself. $\Delta \theta_{i}$ is a random variable uniformly distributed in the interval $[-\eta / 2, \eta / 2]$. Obviously, $\left\langle\theta_{i}(t)\right\rangle_{r}$ is given by:

$$
\tan \left[\left\langle\theta_{i}(t)\right\rangle_{r}\right]=\frac{\left\langle v_{i} \sin \theta_{i}(t)\right\rangle_{r}}{\left\langle v_{i} \cos \theta_{i}(t)\right\rangle_{r}} .
$$

Moreover, in order to measure the degree of consensus of all the particles, an order parameter [6] is introduced as

$$
\phi=\left|\frac{\sum_{i=1}^{N} \vec{v}_{i}}{\sum_{i=1}^{N}\left|\vec{v}_{i}\right|}\right|, \quad 0 \leq \phi \leq 1 .
$$

A larger value of $\phi$ indicates a better consensus, especially when $\phi=1$, all particles move towards the same direction. In the circumstances of high density and low noise, all the particles will definitely approach the consensus state, namely reach the same direction of velocity after finite time steps [7].

Because of the limitation of horizon radius, each particle can only communicate with partial particles within the radius and change its direction according to this local information. Different horizon radii and particle densities will give rise to diverse convergence behaviors and times, which will be systematically investigated later. Without lose of generality, the area is fixed, thus the particle density can be directly represented by the number of particles, $N$.

The simulation results about the convergence time $T$ based on the noisefree Vicsek model $(\eta=0)$ are shown in Fig. 1, where $T$ represents the number 


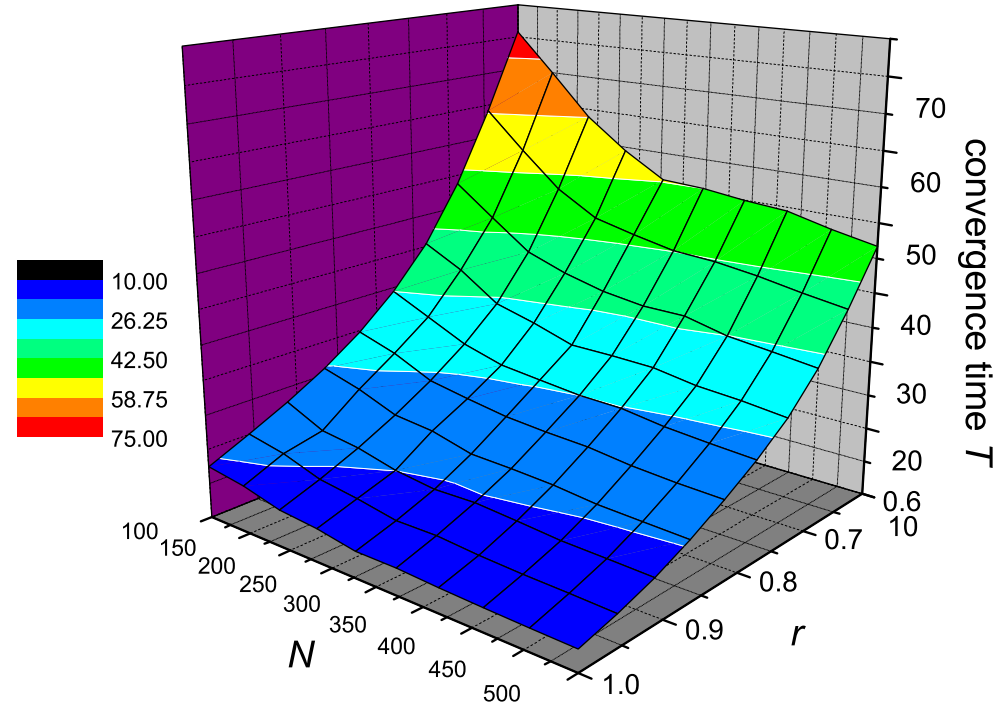

Fig. 1. (color online) Dependence of convergence time $T$ on horizon radius $r$ and the number of particles $N$. In the simulation, all the particles move in a square shaped plane of linear size $L=5$, with a constant speed as $v=0.05$. Control parameters $r$ and $N$, respectively, vary from 0.5 to 1 and from 100 to 500 . The convergence time is obtained from the average over 500 independent runs.

of time steps taken before the order parameter $\phi$ firstly reaches the threshold $\phi_{t}=0.95$. As long as the threshold $\phi_{t}$ is larger enough (close to 1 ), the variance of its specific value will not impact much on the qualitative results shown in this paper. Fig. 1 clearly indicates that the convergence time decreases with the increase of radius and density. This fact can be explained in the following two aspects. (a) Given the density of particles, the larger the horizon radius, the less steps taken to reach synchronization, because at each time step each particle can receive more information from others and thus make the adjustment of velocity more comprehensively, namely more close to the final convergence velocity, than those with shorter radius. (b) Given horizon radius, when the number of particles increases, although the percentage of particles which communicate with a given particle does not increase as well, higher density is also helpful to reduce the convergence time, because the particle is making more integral adjustments at each time step for the increase of the number of other particles inside its horizon (those particles possess different directions of velocity, come from different areas a time step before, bring in information from different areas at latest time step and then pass the information of current adjusted velocity to different areas at the following time step). 


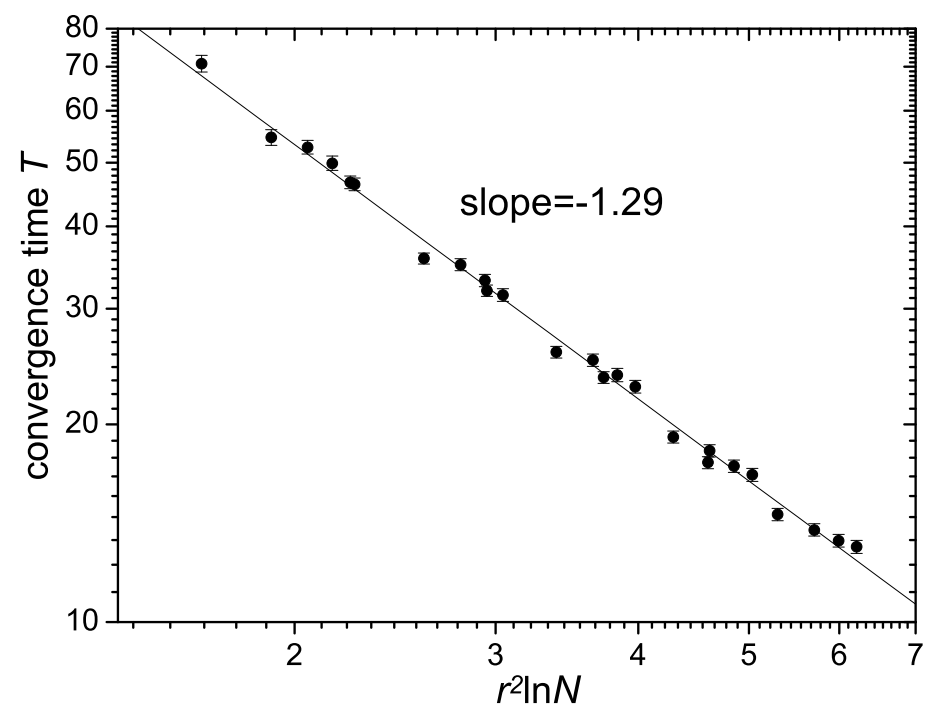

Fig. 2. Convergence time $T$ as a function of $r^{2} \ln N$. The data points can be well fitted linearly in double logarithmic coordinates, with slope and error bars marked in the plot. Each data point is collected from the average over 500 independent runs.

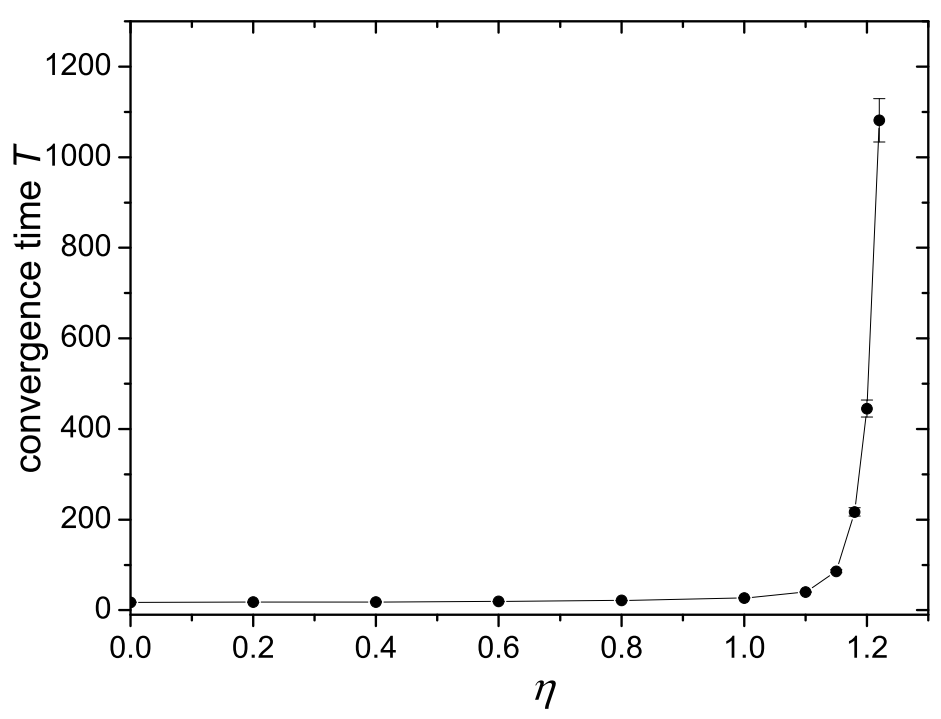

Fig. 3. Convergence time $T$ as a function of the noise $\eta$. We set $r=1.0$ and $N=100$.

Through extensive simulations we eventually come to a numerical conclusion that the convergence time $T$ follows a power law with $r^{2} \ln N$. Fig. 2 shows a numerically approximate relation:

$$
T \sim\left(r^{2} \ln N\right)^{-1.29}
$$


In the case with noise, namely $\eta>0$, the convergence time will increase with the noise strength. In Fig. 3, when $\eta<1.1$, the convergence time increases slightly, while when $\eta>1.1$, the convergence time increases dramatically, especially when $\eta>1.2$, the order parameter can not achieve $\phi_{t}=0.95$, namely with high noise this collective motion can not achieve consensus. This result is quite in according with Fig. 2 in Ref. [6]. Though quantitatively different, the sharp increasing of $T$ (as the increasing of $\phi$ ) can be generally observed for different parameters $\left(r, N, \phi_{t}\right)$.

\section{Fast convergence collective motion with variable speed}

Without taking account of generation and annihilation of particles, the number of particles would be generally fixed. In addition, it might be difficult to accelerate the consensus process by simply changing the horizon radius, for which means higher requirement on both technology and the hardware cost. Considering the speed of particle in collective motion systems can be variable $[13,14]$, we propose a new consensus strategy that achieves improvement in terms of the convergence time compared with the standard Vicsek model.

With limited horizon radius, a particle could only make the judgment by itself from the local information it receives. In a completely chaotic case, although each particle updates its direction to the average one in its local area, this average direction may be far different from the final synchronization direction. Therefore, it should be cautious to act with a comparatively conservative strategy, that is, taking a relatively lower velocity to prevent from the unnecessary change of its position. Here the unnecessary change means that, if a particle changes its position hastily in order to communicate with another group of particles under such chaotic circumstance, it would be sure that such impatient behaviors are of no help. On the contrary, if all the particles choose the conservative strategy of moving with a comparatively lower velocity, then every particle could have sufficient time to communicate with its neighbors, leading to a possibly faster convergence. Only when a certain moving direction is dominant among its neighbors of a particle, it can surely update its direction as that one and take a relatively higher velocity since in that case it is unnecessary for this particle to continue hesitating. Therefore, a particle's velocity should be somehow determined by the degree of its local consensus.

In order to present the degree of local synchronization quantitatively, $\phi_{i}$ is 
introduced, called the local order parameter of consensus for the $i$ th particle:

$$
\phi_{i}=\left|\frac{\sum_{j=1}^{N_{i}} \vec{v}_{i j}}{\sum_{j=1}^{N_{i}}\left|\vec{v}_{i j}\right|}\right|, \quad 0 \leq \phi_{i} \leq 1,
$$

where $N_{i}$ is the number of particles within the horizon radius of the $i$ th particle (including itself). The larger value of $\phi_{i}(t)$, the higher degree of local consensus among the neighbors of the $i$ th particle. Especially when $\phi_{i}(t)=1$, all $i$ 's neighbors move towards the same direction.

Besides, in order to compare with the Vicsek model in terms of the convergence time, we suppose that the absolute speed can vary from 0 to 0.05 . According to the discussion mentioned above, this consensus strategy with variable speed should satisfy:

a) When all particles in $i$ 's neighborhood arrive at an ordered direction as $\phi_{i}(t)=1, v_{i}(t+1)=0.05$;

b) When $\phi_{i}(t)=0$, namely the motions of the particles in $i$ 's neighborhood is completely disorder, $v_{i}(t+1)$ approaches to zero.

Consequently, we set the speed of the $i$ th particle as:

$$
v_{i}(t+1)=v_{\max } e^{\beta\left[\phi_{i}(t)-1\right]},
$$

where $v_{\max }$ is set as 0.05 in this paper. Here $\beta$ is a free parameter. When $\beta=0$ the protocol degenerates to the standard Vicsek model, while for $\beta>0$, a particle will move faster in a better synchronized local circumstance. Indeed, in the present protocol, speed not only determines the position in the next time step, but also serves as a carrier, possessing the information about the local order parameter. The moving direction of the $i$ th particle is also updated following Eq. (4). Note that, when $\phi$ approaches to $1, \phi_{i}$ gets close to 1 as well. Therefore from Eq. (8) we know that the speeds of all particles at this time are all close to 0.05 , which automatically ensure consensus on the absolute speed.

To sum up, the present protocol with adaptive speed can be described as follows:

(1) Determine the initial position and speed of every particle $i$;

(2) Evaluate the local order parameter of each particle, and determine its next direction and speed according to Eqs. (4) and (8);

(3) Calculate the current order parameter $\phi$ of all particles;

(4) Repeat (2) and (3) until $\phi$ approaches to 1. 


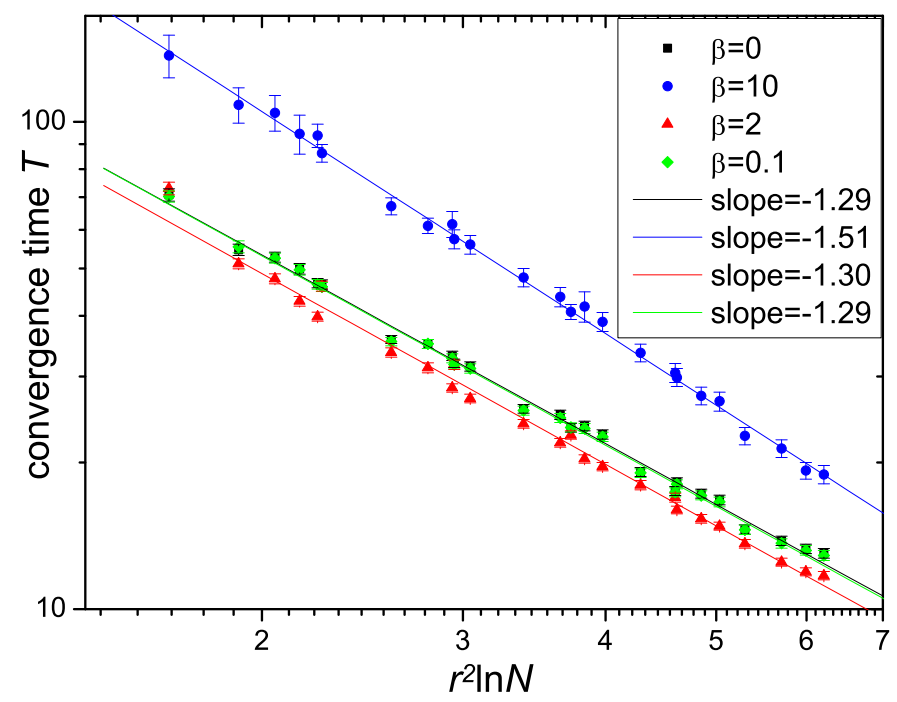

Fig. 4. (color online) Convergence time $T$ as a function of $r^{2} \ln N$ with different $\beta$. The data points can be well fitted linearly in a double logarithmic coordinates, with slopes and error bars marked in the plot. Control parameters $r$ and $N$, respectively, vary from 0.5 to 1 and from 100 to 300 . The convergence time is obtained from the average over 500 independent runs.

In the numerical simulations, we first consider the situation without noise $(\eta=0)$, and still assume that all particles move in a square-shaped plane of linear size $L=5$. The relation between the convergence time $T$ and the horizon radius $r$ and the number of particles $N$ with different $\beta$ is shown in Fig. 4. From this figure, we can see that in this new protocol the convergence time $T$ also has a power function with $r^{2} \ln N$, the same rule as illustrated in section 2. Especially, when $\beta$ is not large enough $(\beta<5)$, the exponents are almost the same, nearly equal to 1.30. More importantly, in Fig. 4 we can find that, compared with the standard Vicsek model, the convergence time can be shortened, which demonstrate the advantage of this modified model in terms of the convergence time.

To further explore this point, we keep the horizon radius $r$ and the number of particles $N$ fixed and try to find out the optimal $\beta$ subject to the shortest convergence time. In Fig. 5, we can see that in all the four curves the optimal $\beta$ is 2 and this optimal $\beta$ is not susceptive to the horizon $r$ and the number of particles $N$. Especially, the convergence time can be highly shortened when the horizon radius $r$ is small.

In Fig. 6 , we can see that when $\beta$ is small (e.g. $\beta=0.1$ ), no matter what is the current speed distribution, the speeds of most particles in the next time will approach to the upper limit 0.05 , resulting in almost the same performance 


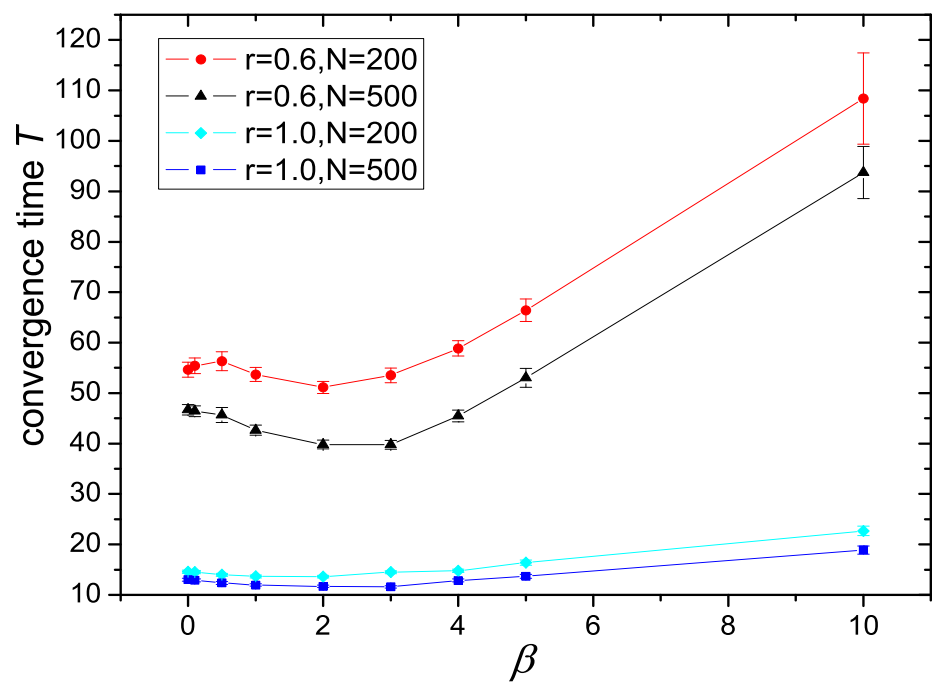

Fig. 5. (color online) Convergence time VS. $\beta$ with different $r$ and $N$.

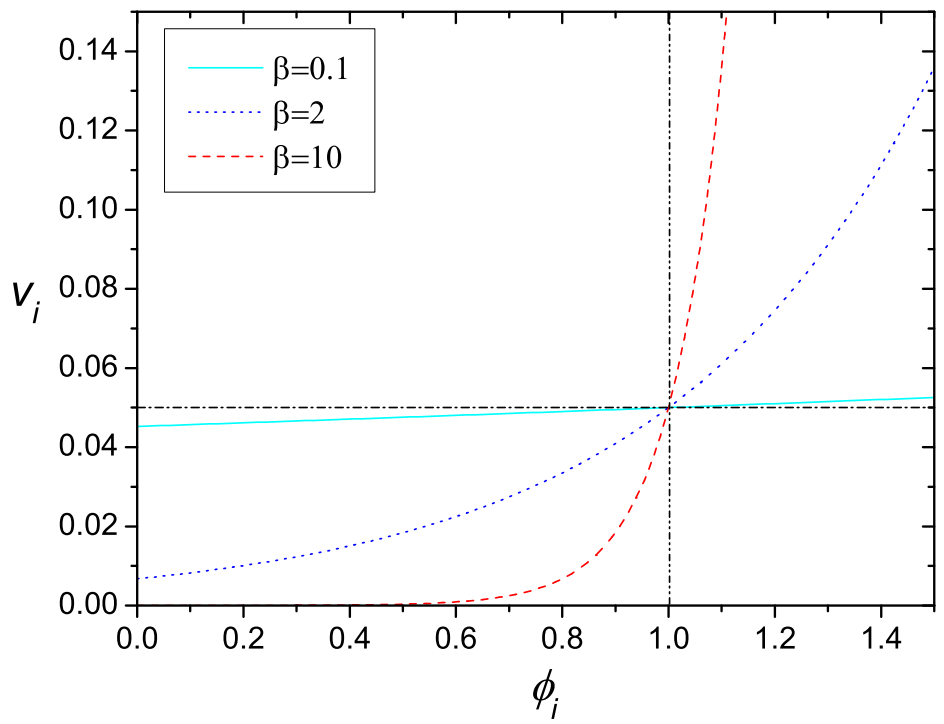

Fig. 6. (color online) Function $v_{i}\left(\phi_{i}\right)=0.05 e^{\beta\left[\phi_{i}-1\right]}$ for $\beta=0.1,2$ and 10.

as the standard Vicsek model; besides, when $\beta$ is too large (e.g. $\beta=10$ ), the speeds of particles will tend to be 0 , thus even when the neighbors of a given particle is relatively ordered, it still keeps rest and therefore cannot intercommunicate with others, thus resulting in a extremely long convergence time. 
For a proper value of $\beta$ (e.g. $\beta=2$ ), when a particle has a large local order parameter, it will have a great confidence on its current direction, thus leaving the area with a high speed and using the information (speed direction) to influence others; Otherwise, it will cast doubt on whether the current direction is up to the demand of consensus, and in order to avoid misguiding others with its unconfirmed direction, it will march on with a relatively slow speed.

\section{Conclusions}

The collective dynamics of intelligent agents is not only the extensive phenomena in nature, but also an important problem required in-depth investigation in engineering. Most of the previous studies concentrated on the depiction and modeling of the swarm itself. The systematical analysis about the convergence time were rarely reported. In this paper we have studied the relationship between the convergence time and the particle density as well as the horizon radius in the Vicsek model, and found that the convergence time $T$ has a power function of $r^{2} \ln N$. Furthermore, we have designed a motion protocol variable speed, in which the speed is not only a moving parameter, but also can deliver some information about its situation of local synchronization. Under such protocol, the convergence process can be remarkably accelerated compared with the standard Vicsek model.

\section{Acknowledgements}

We acknowledge Ming Zhao, Luo-Luo Jiang and Da-Jie Zeng for their assistances for manuscript preparation. This work is partially supported by the

National Natural Science Foundation of China under Grant Nos. 10472116 and 10635040, as well as the 973 Project 2006CB705500. H.T.Z. acknowledges the National Science Foundation of China under Grant No. 60704041.

\section{References}

[1] E. Bonabeau, G. Theraulaz, V. Fourcassie and J.L. Deneubourg, Phys. Rev. Lett. 57 (1998) 4568.

[2] E. Ben-Jacob, I. Cohen and D.L. Gutnick, Annual Review of Microbiology 52 (1998) 779.

[3] S. Huang, C.P. Brangwynne, K.K. Parker and D.E. Ingber, Cell Motility and the Cytoskeleton 61 (2005) 201. 
[4] S. Hubbard, P. Babak, S.T. Sigurdsson and K.G. Magnusson, Ecological Modeling 174 (2004) 359.

[5] A.V. Savkin, IEEE Trans. Autom. Control. 49 (2004) 981.

[6] T. Vicsek, A. Czirók, E. Ben-Jacob, I. Cohen and O. Shochet, Phys. Rev. Lett. 75 (1995) 1226.

[7] A. Jadbabaie, J. Lin and A.S. Morse, IEEE Trans. Autom. Control. 48 (2003) 988.

[8] G. Grégoire and H. Chaté, Phys. Rev. Lett. 92 (2004) 025702.

[9] M. Aldana, V. Dossetti, C. Huepe, V.M. Kenkve and H. Larradlde, Phys. Rev. Lett. 98 (2007) 095702.

[10] I.D. Couzin, J. Krause, R. James, G.D. Ruxton and N.R. Franks, J. Theor. Biol. 218 (2002).

[11] I.D. Couzin, J. Krause, N.R. Franks and S.A. Levin, Nature 433 (2005) 513.

[12] J. Toner, Y.H. Tu and S. Ramaswamy, Annals of Physics 318 (2005) 170.

[13] W. Li and X.F. Wang, Phys. Rev. E 75 (2007) 021917.

[14] W. Li, H.T. Zhang, M.Z.Q. Chen, and T. Zhou, Phys. Rev. E 77 (2008) 021920. 